Pediatr Crit Care Med 2007;8:207-211). At Starship Children's Hospital, Auckland, NZ, 72 children $(<12$ months old) were admitted to the PICU with pertussis from 1991-2003. Apnea and cough were presenting symptoms in $83 \%, 4$ died and 6 had neurodevelopmental problems. (Surridge J et al. Arch Dis Child 2007;92:970-975). In unimmunized populations, pertussis is a serious disease with complications, principally respiratory.

\title{
REVERSIBLE VALPROATE HEPATOTOXICITY AND ASSOCIATED MITOCHONDRIAL DISEASE
}

A 2-year-old boy with seizures complicated by reversible valproate-induced hepatic failure was subsequently found to have mitochondrial polymerase $\mathrm{g}$ gene (POLG1) mutations typical of Alpers-Huttenlocher disease, in a study at Newcastle University, UK. Brain MRI showed abnormal white matter signal in occipital and medial temporal lobes bilaterally. After discontinuing sodium vaiproate and substituting levetiracetam, liver function returned to normal over a 6-month period. Sequencing of $P O L G 1$ is recommended in children with valproate-induced hepatic failure, and prior to commencing sodium valproate in young children ( $<3$ years old $)$ with aggressive focal epilepsy. Serum lactate, ammonia and liver function should be closely monitored in young children treated with valproate. (McFarland R, Hudson G, Taylor RW et al. Reversible valproate hepatotoxicity due to mutations in mitochondrial DNA polymerase g (POLG1). Arch Dis Child Feb 2008;93:151-153). (Respond: Dr Robert McFarland, Mitochondrial Research Group, School of Neurology and Psychiatry, $4^{\text {th }}$ Floor, Medical School, Framlington Place, Newcastle University, Newcastle NE2 4HH, UK).

COMMENT. The syndrome of diffuse progressive degeneration of the cerebral gray matter was first described by Alpers in 1931. Ford (1951) differentiated infantile and juvenile types and reported familial cases. Huttenlocher et al (1976) described coincident hepatic cirrhosis. Egger et al (1987) reported 13 cases treated at the Hospital for Sick Children, Great Ormond Street, London, 4 having received sodium valproate. A genetically determined, autosomal recessive, metabolic cause was suggested. Bicknese et al (1992) from Washington University, St Louis, MO, reported 6 patients with Alpers-Huttenlocher syndrome, 4 taking valproic acid (VPA), 2 of whom had a sibling with the same syndrome but no exposure to VPA. Siblings receiving VPA survived only 3 and 5 months after onset of seizures, whereas those not receiving VPA lived for 7 to 16 months. These authors proposed that many of the cases of VPA-associated hepatotoxicity represent undiagnosed hepatocerebral degeneration, Huttenlocher variant of Alpers' syndrome. In 2004, Naviaux et al described POLG mutations associated with Alpers' syndrome and mitochondrial DNA depletion. Patients with the syndrome treated with VPA have developed an irreversible liver failure and neurologic decline. The present case is exceptional, having a more favorable outcome.

A 17-year-old adolescent girl with Juvenile Alpers Disease is reported from the University of Otago, Wellington, NZ and other centers. (Wiltshire E et al. Arch Neurol Jan 2008;65:121-124). She presented with clusters of occipital seizures and clumsiness, and showed progressive memory impairment, slurred speech, and hemiparesis. She died of respiratory failure, cerebral degeneration, and liver necrosis. Mutational analysis of POLGI showed 2 novel mutations, similar to the abnormality in infantile Alpers disease. 